

\title{
Utilisation des processus décisionnels de Markov pour l'aide à la maîtrise d'une maladie animale
}

Anne-France Viet, Laurent Jeanpierre, Maroua Bouzid, Abdel-Illah Mouaddib

\section{To cite this version:}

Anne-France Viet, Laurent Jeanpierre, Maroua Bouzid, Abdel-Illah Mouaddib. Utilisation des processus décisionnels de Markov pour l'aide à la maîtrise d'une maladie animale. Revue des Sciences et Technologies de l'Information - Série RIA : Revue d'Intelligence Artificielle, 2013, 27 (4-5), pp.471-492. 10.3166/ria.27.471-492 . hal-00975865

\section{HAL Id: hal-00975865 https://hal.science/hal-00975865}

Submitted on 15 Apr 2014

HAL is a multi-disciplinary open access archive for the deposit and dissemination of scientific research documents, whether they are published or not. The documents may come from teaching and research institutions in France or abroad, or from public or private research centers.
L'archive ouverte pluridisciplinaire HAL, est destinée au dépôt et à la diffusion de documents scientifiques de niveau recherche, publiés ou non, émanant des établissements d'enseignement et de recherche français ou étrangers, des laboratoires publics ou privés. 


\title{
Utilisation des processus décisionnels de Markov pour l'aide à la maîtrise d'une maladie animale
}

\author{
Viet A.-F., ${ }^{1,}$, Jeanpierre L. ${ }^{2}$, Bouzid M. ${ }^{2}$, Mouaddib A.-I. ${ }^{2}$ \\ 1. INRA, LUNAM Université, Oniris, UMR1300 BioEpAR, \\ CS 40706, F-44307 NANTES cedex 3, France \\ anne-france.viet@oniris-nantes.fr \\ 2. Normandie Univ; UNICAEN, GREYC; ENSICAEN, GREYC; CNRS, UMR6072 \\ GREYC \\ CS 5186, F-14032 CAEN, France \\ \{laurent.jeanpierre; maroua.bouzid-mouaddib ; abdel- \\ illah.mouaddib\}@unicaen.fr
}

RESUME. La maîtrise d'une maladie animale non réglementée est à l'initiative des éleveurs et est parfois incitée par des organisations professionnelles pour améliorer la situation sanitaire ou économique d'une zone. Une organisation peut donc proposer à ses membres des recommandations pour maîtriser la maladie, ces recommandations pouvant varier au cours du temps selon l'évolution de la situation épidémiologique dans la zone. L'enjeu est donc de pouvoir proposer des outils d'aide à la prise de décision et d'évaluer a priori l'impact des décisions proposées sur la propagation d'un agent pathogène en termes épidémiologiques (prévalence de la maladie) et économiques. Dans cet article, nous évaluons l'apport des processus décisionnels de Markov (MDP). Nous proposons un modèle de propagation intertroupeaux où une action de maîtrise est recommandée par un décideur collectif pour optimiser le coût de la maladie et de sa maîtrise au niveau du groupe. Nous supposons que le décideur collectif connaît la proportion d'éleveurs qui vont suivre sa recommandation. L'utilisation d'un MDP intégrant un modèle épidémiologique permet d'indiquer à chaque pas de temps s'il faut faire une recommandation ou non selon la situation épidémiologique. La stratégie obtenue consiste en des recommandations non systématiques. Bien que l'objectif soit d'optimiser les coûts, la prévalence dans la zone est aussi diminuée. La définition d'une stratégie adaptative est un avantage de notre approche qui permet de proposer des stratégies non classiquement proposées et étudiées.

ABSTRACT: Control of unregulated animal diseases depends on farmers but is often encouraged by professional organizations to enhance the health status or the economy in the area. Organizations can offer its members incentives to control the disease. These incentives may change over time depending on the epidemiological situation. The challenge is to be able to offer tools for making decisions and assessing a priori the impact of proposed decisions on 
the spread of a pathogen in epidemiological (prevalence) and in economic terms. In this paper, we evaluate the contribution of Markov Decision Processes (MDP): We propose a model of between-herds spread when a control action is prompted by a collective decision maker to optimize the cost of the disease and of its control at the group level. We suppose the efficiency of the advice is known. Then, an epidemiological MDP model can suggest at each time whether advice should be given or not. Resulting strategy is non-systematic. Although the goal was to decrease costs, we observe the prevalence decreases also. Here, an adaptive strategy is an advantage of our approach because these strategies are often not studied.

Mots-CLES : Décision collective, Épidémiologie, Politique, Santé animale, MDP

KEYWORDS: Collective decision, epidemiology, policy, animal health, MDP

DOI:10.3199/JESA.45.1-n @ Lavoisier 2012 AR_DOI

\section{Introduction}

La nécessité de prévenir et de maîtriser la propagation des maladies animales est devenue un sujet d'intérêt en Europe à partir du $18^{\text {eme }}$ siècle (Thrusfield, 1986). La plupart des maladies animales contagieuses ayant de graves conséquences sont maîtrisées ou éradiquées entre la fin du $19^{\text {ème }}$ et le milieu du $20^{\text {ème }}$ siècle dans les pays développés (Thrusfield, 1986). Les principales maladies animales transmissibles induisant des risques pour la santé publique ou la sécurité des aliments, ou ayant des conséquences socio-économiques marquées ont été répertoriées par l'OIE (Organisation Mondiale de la Santé Animale). La prévention et le contrôle de ces maladies sont des objectifs au niveau international : elles sont dites réglementées. Mais il existe d'autres maladies ayant actuellement des conséquences économiques sans être réglementées. Les éleveurs peuvent cependant décider de mettre en place des actions de maîtrise. Des organisations professionnelles proposent parfois des recommandations ou incitations ou des approches collectives de maîtrise d'une maladie. Le choix d'une action de maîtrise est raisonné à partir de l'évaluation préalable de leur efficacité et de la situation épidémiologique. L'enjeu est de pouvoir proposer des outils d'aide à la prise de décisions et d'évaluer a priori l'impact des décisions proposées sur la propagation d'un agent pathogène en termes épidémiologiques (prévalence de la maladie) et économiques. Ces prises de décisions se font dans un contexte d'incertitude quant à l'évolution de la maladie. Des décisions sont alors prises et réajustées régulièrement (décisions séquentielles) en tenant compte de la situation épidémiologique au moment de chaque prise de décision (adaptation). Les modèles décisionnels de Markov (MDP) sont donc particulièrement adaptés à la résolution de ce type de problème.

La prise de décision séquentiellement et de manière adaptative est courante en écologie (Chadès et al., 2008 ; Chadès et al., 2011 ; Johnson et al., 2011 ; Moore et al., 2011). En santé animale, contrairement aux questions qui se posent en écologie, la décision au niveau d'un groupe d'élevages pour la maîtrise d'une maladie non 
réglementée n'est pas centralisée. Les éleveurs prennent leur décision et tiennent compte des recommandations faites par des organisations collectives. Rat-Aspert et Fourichon (2010) formalisent ces prises de décision individuelles et simulent l'effet de différentes incitations. A notre connaissance, les approches se positionnant au niveau collectif pour l'optimisation sont plus rares. En épidémiologie animale, elles ont été développées pour des maladies réglementées, et donc sans décision des éleveurs (Ge et al., 2010). Elles ont aussi été proposées en santé humaine comme décrit dans (Merl et al., 2009 ; Yaesoubi et Cohen, 2011).

L'objectif de cet article est de présenter un problème de décision en santé animale pour des maladies non réglementées et une application des méthodes d'optimisation MDP. Dans une première partie, nous présentons le contexte en santé animale puis le problème de la coordination de stratégies individuelles au sein d'un groupement d'élevages avec sa formalisation et un exemple de résultats. Nous finirons par une discussion sur les apports de l'intelligence artificielle pour notre problématique et ses extensions.

\section{Problématique en santé animale}

Pour les maladies animales non réglementées, les éleveurs n'ont pas l'obligation de mettre en place des actions de maîtrise dans leur exploitation. Chaque éleveur choisit de mettre en place ou non des actions de maîtrise en se basant sur différents critères (économique, zootechnique, ...) et sur sa perception du risque (Klein et al, 2007). Les critères et la perception diffèrent d'un éleveur à un autre. Or le choix d'une stratégie de maîtrise dans une exploitation va influencer la propagation de la maladie dans cette exploitation, et donc le risque de transmission aux autres (par voisinage ou par achat d'animaux) ainsi que la propagation dans la région. Des organisations professionnelles proposent parfois des recommandations ou des approches collectives de maîtrise d'une maladie. En effet, une amélioration de la situation sanitaire dans une région pour certains agents pathogènes transmissibles peut améliorer la compétitivité des exploitations concernées. Elles cherchent donc à faire des recommandations parfois en proposant des incitations financières pour limiter la propagation de la maladie (prévalence) ou son aspect économique. Ces recommandations peuvent varier au cours du temps selon l'évolution de la situation épidémiologique dans la région.

L'impact d'actions décidées au niveau collectif sur la propagation est souvent étudié par modélisation pour fournir aux organisations des aides à la prise de décision. Classiquement, les stratégies étudiées consistent en une seule action qui est appliquée sur toute une période. Très peu d'études considèrent des actions différentes au cours du temps. Souvent ces études portent sur l'effet d'une action à chaque apparition d'un nouveau cas (Baker et al., 2009; Tildesley et al., 2006; Keeling et al., 2003; Ferguson et al., 2001). Pour la propagation d'un pathogène dans une population d'animaux d'élevage, la définition d'une stratégie dont l'action à mettre en place dépend de la prévalence de l'infection peut être définie aux dires d'experts (par exemple dans Seegers et al., 2006). Pour déterminer la meilleure 
action à un instant donné en fonction de la situation épidémiologique, différentes approches ont été retenues : arbre de décisions (Rat-Aspert et Fourichon., 2010), simulation des différents comportements possibles dans le futur (Merl et al., 2009) ou par d'autres méthodes d'optimisation (Kristensen, 2003; Toft et al., 2005; Kobayashi et al., 2007; Ge et al., 2010). Ces approches à base de planification permettent de proposer des stratégies innovantes au contraire des approches à base de simulation qui ne permettent que d'évaluer des stratégies pré-définies (par exemple par des experts).

Les processus décisionnels de Markov (MDP) sont une approche d'optimisation utilisée pour résoudre des problèmes de prise de décision séquentielle (à chaque pas de temps) dans un environnement incertain, ce qui correspond à notre problème. Ils permettent le calcul d'une politique donnant l'action à mettre en place selon l'état du système (Puterman, 2005). Ils sont utilisés en planification en robotique (par exemple : Boussard et al., 2007) ou en gestion des ressources (par exemple : Forsell et al., 2012), ou en écologie (par exemple : Chadès et al., 2008 ; Chadès et al., 2011 ; Johnson et al., 2011 ; Moore et al., 2011). Cette approche a été utilisée en santé animale pour une maladie réglementée ( $\mathrm{Ge}$ et al, 2010), en épidémiologie humaine (Galvani et al., 2007), en épidémiologie végétale (Peyrard et al., 2007) et en épidémiologie animale (Viet et al., 2012). Elle permet de tenir compte de l'incertitude sur la propagation de la maladie pouvant induire plusieurs situations épidémiologiques possibles à un même instant. De plus, les décisions à chaque instant dépendent de la situation épidémiologique observée et non de la situation moyenne attendue.

\section{Coordination dans un groupe d'élevages}

\subsection{Présentation}

On considère une structure regroupant $\mathrm{N}$ troupeaux. Cette structure peut faire des recommandations aux éleveurs de son groupe pour qu'ils protègent les troupeaux non infectés afin de limiter l'impact économique ou épidémiologique de la propagation d'une maladie animale dans la structure.

On suppose que chaque troupeau a un statut vis à vis de la maladie considérée, complètement connu au cours du temps : sensible ou infecté. La transmission de la maladie se fait par contact entre troupeaux de la structure (incluant les achats) et par contact avec d'autres troupeaux à l'extérieur de la structure (risque constant de s'infecter). Pour la maîtrise de la maladie, il est possible de réduire les contaminations en protégeant les troupeaux sensibles (biosécurité, vaccination).

Le décideur collectif va chercher à optimiser le coût de la maladie et de sa maîtrise pour l'ensemble de la structure. Pour les coûts de circulation de la maladie, on suppose que chaque troupeau infecté a un coût (pertes liées à la maladie). Pour les coûts liés à la maîtrise, on suppose que lorsque la protection d'un troupeau sensible est retenue, elle induit un coût pour l'organisation proportionnel au nombre 
de troupeaux protégés. On suppose de plus que le collectif a des coûts supplémentaires liés à la diffusion de l'information pour inciter à la protection.

Dans ce modèle, on ne représente pas explicitement la prise de décision individuelle pour éviter une explosion de la taille du modèle. Pour des maladies non réglementées, les décisions collectives n'ont pas de caractère obligatoire et donc elles ne sont pas toujours suivies par les éleveurs. Ce n'est donc pas une action centralisée. Par conséquent, il est nécessaire de représenter l'interaction décision collective et décisions individuelles. Pour limiter la complexité du modèle, nous avons donc choisi de représenter, lorsqu'il y a recommandation, l'effet des décisions individuelles sur la transmission de la maladie. Pour simplifier, on suppose que la proportion d'éleveurs qui va retenir l'action de protéger son troupeau suite à la recommandation est connue et fixe.

Le décideur collectif va chercher à identifier quand il doit faire une recommandation pour que la protection des troupeaux sensibles soit mise en place selon la situation épidémiologique actuelle et les évolutions futures possibles. Classiquement on considère que certains éleveurs ayant un troupeau sensible n'auront pas le comportement attendu. Certains peuvent protéger sans recommandation du collectif alors que d'autres ne protégeront pas même avec la recommandation (et incitation financière). Le pourcentage de troupeaux qui ne suivront pas la recommandation est supposé connu par le décideur et constant au cours du temps. Chaque année, le décideur fait des recommandations ou non aux éleveurs de la structure pour qu'ils protègent les troupeaux non infectés.

\subsection{Modèle formel}

Pour l'optimisation, on définit un processus décisionnel de Markov intégrant un modèle épidémiologique. Notre modèle décrit la dynamique temporelle de la propagation de la maladie et des décisions. Il est stochastique afin de décrire la variabilité de la propagation dans l'ensemble des troupeaux considérés et de tenir compte de cette variabilité dans la prise de décision. Dans un but de généralisation, le modèle est développé avec un pas de temps mensuel mais les recommandations et la prévention ne sont faites qu'une fois par an. Cela permettra d'étendre ultérieurement le modèle avec des actions plus complexes (actions différentes selon le mois par exemple). Le modèle est markovien et à compartiments. Un compartiment contient tous les troupeaux ayant un même statut vis à vis de la maladie.

Avant de présenter le modèle épidémiologique et le MDP correspondant, nous allons rappeler le formalisme des MDP. Pour plus de détails, voir (Garcia, 2008).

\subsubsection{Préliminaires}

Un MDP est défini par un tuple $<\mathrm{E}, \mathrm{A}, \mathrm{T}, \mathrm{R}>$ tel que

- $\quad$ E est l'ensemble fini d'états du modèle, chaque état pouvant être atteint. 
- A est l'ensemble fini des actions possibles.

- $\quad \mathrm{T}: \operatorname{ExAxE} \rightarrow[0,1]$ est la fonction de transition. T(s,a,s') est la probabilité de devenir $s^{\prime}$ lorsqu'on utilise l'action $a$ dans l'état $s$. Cette probabilité représente l'incertitude sur les conséquences de l'action $a$.

- $\quad \mathrm{R}: \operatorname{ExAxE} \rightarrow \mathrm{R}$ est la fonction de récompense. $\mathrm{R}\left(\mathrm{s}, \mathrm{a}, \mathrm{s}^{\prime}\right)$ est la récompense après avoir utilisé l'action $a$ dans l'état $s$ qui a conduit le système dans l'état $s$ '. Une récompense négative permet d'assigner un coût à une action.

En résolvant un MDP, on obtient une politique qui indique quelle est l'action optimale à faire dans chaque état. Dès lors, avec une seule et même politique, la séquence d'action (ou de recommandation dans notre cas) qui sera finalement choisie dépendra de l'évolution "réelle" du processus. Une politique définit donc une sorte de plan adaptatif et optimal en moyenne. Cette politique $\pi$ est donc une fonction de $\mathrm{E}$ vers $\mathrm{A}$ donnant l'action à retenir pour chaque état de $\mathrm{E}$. La valeur de la politique $\pi$ à un horizon fixé $h$ est l'espérance de la somme des récompenses pondérées pour les $h$ prochains pas de temps :

$$
V^{\pi}(s)=E\left[\sum_{t=1}^{h} \gamma^{t} R\left(s_{t}, a_{t}, s_{t+1}\right) \mid \pi\right]
$$

avec $\gamma$ le facteur d'atténuation $(0 \leq \gamma \leq 1)$ et $S_{t}$ l'état atteint à l'instant $t$ en suivant la politique $\pi$ à partir de l'état $\mathrm{s}$. Le facteur d'atténuation $\gamma$ est le poids à l'instant $\mathrm{t}$ d'une unité de récompense reçue à $\mathrm{t}+1$. Il correspond au taux d'actualisation classiquement utilisé en économie. Résoudre un MDP revient à obtenir la politique optimale $\pi^{*}$. Cette politique est celle qui maximise $V^{\pi}(s) \forall s \in E$. Des algorithmes existent pour calculer exactement cette politique optimale $\pi^{*}$, en particulier Value Iteration et Policy Iteration (Puterman, 2005). On distinguera les horizons finis où la politique dépend du temps des horizons infinis où la politique est stationnaire.

\subsubsection{Modèle épidémiologique au niveau individuel}

Dans le modèle épidémiologique, trois compartiments ont été considérés : Sensible (S), Infecté (I) et Protégé (P). On suppose que la maladie n'induit pas d'immunité du troupeau et donc qu'un troupeau I redevient $S$ après la fin de l'infection (fig 1). La protection est réalisée une fois par an et induit une protection contre l'infection pendant environ 1 an. 


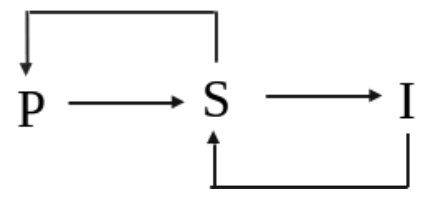

Figure 1. Représentation schématique des transitions entre statuts individuels ( $P$ : protégé, $S$ : sensible, $I$ : infecté).Les probabilités de transitions entre statuts dépendent de l'action du collectif.

D'un point de vue individuel (Fig. 1), un troupeau S peut s'infecter (I) ou, 1 fois par an, être protégé $(\mathrm{P})$. Un troupeau $\mathrm{P}$ perd sa protection au cours du temps (il redevient $S$ ). Un troupeau I peut guérir naturellement (il redevient $S$ ). La probabilité qu'un troupeau $\mathrm{S}$ soit protégé $\mathrm{p}(\mathrm{S}->\mathrm{P})$ dépend de l'action du décideur collectif (recommandation ou non). Les transitions au niveau individuel sont données dans les équations (équation 1)

$$
\left\{\begin{array}{c}
p(S \rightarrow P)=\alpha \\
p(S \rightarrow I)=(1-\alpha) \frac{\beta \cdot c}{N}+p \text { Out } \\
p(S \rightarrow S)=1-p(S \rightarrow P)-p(S \rightarrow I) \\
p(I \rightarrow S)=\eta \\
p(I \rightarrow I)=1-p(I \rightarrow S) \\
p(P \rightarrow S)=\delta \\
p(P \rightarrow P)=1-p(P \rightarrow S)
\end{array}\right.
$$

avec $\alpha$ la proportion de troupeaux où la protection est mise en place correctement (cette proportion est non nulle 1 fois par an mais sa valeur varie selon s'il y a recommandation du collectif ou non) $; \beta$ le taux de transmission qui est multiplié par la proportion de troupeaux infectés dans la structure (forme fréquence-dépendante Bégon et al., 2002), c le nombre de troupeaux infectés dans la structure, $\mathrm{N}$ le nombre total de troupeaux, pOut la probabilité d'une contamination par l'extérieur, $\eta$ la proportion par mois de troupeaux I qui redeviennent sensibles et $\delta$ la proportion par mois de troupeaux $\mathrm{P}$ qui ne sont plus protégés. Les valeurs des paramètres sont données dans le tableau 1. 
Tableau 1. Valeurs des paramètres du modèle (par mois)

\begin{tabular}{|c|c|}
\hline Paramètres & Valeurs \\
\hline Taux de transmission $(\beta)$ & 0,1 \\
\hline Probabilité d'une contamination par l'extérieur (pOut) & 0,005 \\
\hline Probabilité de passer de I à S ( $\eta)$ & 0,02 \\
\hline Probabilité de passer de S à P (1 fois par an) - sans recommandation $(\alpha)$ & 0,05 \\
\hline Probabilité de passer de S à P (1 fois par an) - avec recommandation $(\alpha)$ & 0,75 \\
\hline Probabilité de passer de P à S $(\delta)$ & 0,02 \\
\hline Coût de la protection $(\mathrm{Cp})$ & -9 \\
\hline Coût de la maladie $(\mathrm{Cm})$ & -1 \\
\hline Coût fixe lié à la mise en place de la recommandation $(\mathrm{Cf})$ & -25 \\
\hline
\end{tabular}

\subsubsection{Processus Décisionnel de Markov}

\subsubsection{Espace d'états}

L'évolution du système est décrite par l'évolution du nombre de troupeaux dans chaque statut (S, I, P). Le pas de temps du modèle est d'un mois. Pour introduire le fait que la protection n'est possible qu'une fois par an en respectant l'hypothèse Markovienne, il faut mémoriser dans l'état du système le mois où l'on se trouve (ti avec $0<=i<=11$ ). Cette information va conduire à une augmentation du nombre d'états dans le système mais va permettre de n'autoriser la recommandation et la protection qu'au mois $\mathrm{t} 0$. La généralisation du modèle à d'autres types d'action sera discutée ultérieurement.

L'ensemble des états du système correspond à l'ensemble des couples (ti,s) avec :

- ti : le mois en cours. On considère 12 indicateurs ti qui permettent de noter le mois dans l'année.

- $\mathrm{s}$ : l'état de la population qui correspond à l'ensemble des répartitions des $\mathrm{N}$ troupeaux dans les 3 statuts de troupeaux S, I et P.

Dans notre modèle, l'espace d'états à un mois donné $t$ correspond à l'ensemble des répartitions des troupeaux parmi les 3 statuts individuels vis à vis de l'infection $(\mathrm{S}, \mathrm{I}, \mathrm{P})$. Avec 3 troupeaux, on a $\mathrm{E}=\{(3,0,0),(2,1,0),(2,0,1),(1,2,0),(1,0,2),(1,1,1)$, $(0,3,0),(0,2,1),(0,1,2),(0,0,3)\}$ où $(\mathrm{x}, \mathrm{y}, \mathrm{z})$ sont respectivement le nombre de troupeaux dans les statuts $\mathrm{S}$, I et P. La taille de l'espace d'états est donné par : 


$$
|E|=12 * C_{N+2}^{2}=\frac{12 *(N+2)(N+1)}{2}
$$

où $C_{n}^{p}$ est le coefficient binomial donnant le nombre de combinaison de $\mathrm{p}$ éléments parmi n. Attention, l'espace d'états croît exponentiellement avec la taille de la population (tableau 2). Dans la suite, nous considérerons une population de $\mathrm{N}=100$ troupeaux.

Tableau 2. Taille de l'espace d'états $|E|$ en fonction du nombre de troupeaux donné par l'équation (2)

\begin{tabular}{|c|c|}
\hline $\mathrm{N}$ & Taille de l'espace d'états \\
\hline 50 & 15912 \\
\hline 100 & 61812 \\
\hline 200 & 243612 \\
\hline 300 & 545412 \\
\hline 400 & 967212 \\
\hline 500 & 1509012 \\
\hline
\end{tabular}

\subsubsection{Actions possibles}

L'ensemble des actions disponibles est $\{\mathrm{Ne}$ rien faire; Recommander la protection\}. Attention, l'action « Recommander la protection » n'est pas disponible pour les états avec un t différent de t0.

\subsubsection{Transitions}

Les transitions entre états du système sont fonction des actions. Elles sont calculées à partir de la matrice des transitions individuelles (i.e. pour un troupeau), en considérant l'ensemble des transitions possibles au niveau de la population totale via des distributions multinomiales comme dans (Viet et al., 2012). Pour un état du système $(t,(k, i, j))$, la transition vers l'état $(t+1,(l, m, n))$ va dépendre du nombre de troupeaux qui passent d'un statut à un autre et de l'action considérée. Pour simplifier la présentation, on raisonne à t fixé dans la suite. Le passage de l'état $(\mathrm{k}, \mathrm{i}, \mathrm{j})$ à l'état $(\mathrm{l}, \mathrm{m}, \mathrm{n})$ correspond à différents ensembles de changements individuels de statuts. Par exemple, la transition de $(1,2,0)$ à $(2,1,0)$ à un instant où il n'y a pas de protection des troupeaux peut être la conséquence soit (i) d'un changement de I à S pour 1 troupeau, les autres restant dans le même statut ou (ii) à la fois d'un mouvement de 1 troupeau de $\mathrm{S}$ à $\mathrm{I}$ et des mouvements de 2 troupeaux de I à $\mathrm{S}$. Comme les troupeaux ne sont pas individualisés dans notre modèle, il y a 2 
mouvements possibles : $2=C_{2}^{1}$ dans la situation (i) selon quel troupeau I change de statut et $1=C_{2}^{2}$ dans la situation (ii). On a donc à $\mathrm{t} \neq \mathrm{t} 0$ :

$T((1,2,0), a,(2,1,0))=\left(C_{2}^{1} P(I \rightarrow S) P(I \rightarrow I)\right)\left(C_{1}^{1} P(S \rightarrow S)\right)+\left(C_{2}^{2} P(I \rightarrow S)^{2}\right)\left(C_{1}^{1} P(S \rightarrow I)\right)$

En suivant ce même raisonnement, les transitions au niveau du groupe sont alors calculées en considérant toutes les transitions individuelles compatibles avec le changement d'état au niveau du groupe et en utilisant des coefficients multinomiaux. A $\mathrm{t} \neq \mathrm{t} 0$, la transition $T((h, i, j), a,(l, m, n))$ de $(\mathrm{h}, \mathrm{i}, \mathrm{j})$ à $(1, \mathrm{~m}, \mathrm{n})$ est donnée par

$$
\sum_{u=0}^{h} \sum_{v=0}^{i} \sum_{w=0}^{j} \delta_{\left\{\begin{array}{c}
h+v+w-u=l \\
i+u-v=m \\
j-w=n
\end{array}\right.}\left[\begin{array}{c}
\left(C_{h}^{u} p(S \rightarrow I)^{u} p(S \rightarrow S)^{h-u}\right) \\
*\left(C_{i}^{v} p(I \rightarrow S)^{v} p(I \rightarrow I)^{i-v}\right) \\
*\left(C_{j}^{w} p(P \rightarrow S)^{w} p(P \rightarrow P)^{j-w}\right)
\end{array}\right]
$$

où $\left\{\begin{array}{c}\text { cond }_{I} \\ \cdots \\ \text { cond }_{n}\end{array}\right\}$

est la fonction de Dirac, égale à 1 quand les conditions cond_l, ..., cond_n $n$ sont toutes satisfaites et 0 sinon, $C_{n}^{p}$ est le coefficient binomial pour $\mathrm{p}$ éléments parmi $\mathrm{n}, u$ le nombre de troupeaux $\mathrm{S}$ devenant $\mathrm{I}, v$ le nombre de troupeaux I devenant $\mathrm{S}$ et $w$ le nombre de troupeau $\mathrm{P}$ devenant $\mathrm{S}$. En ne considérant que les états vérifiant la fonction de Dirac, l'équation (3) se simplifie en:

$T((h, i, j), a ; l, m, n)=\left\{\begin{array}{c}B s i\left\{\begin{array}{c}n \leq j \\ m \leq i+h \\ l \geq j-n \\ l \leq j-n+h+i\end{array}\right. \\ 0 \text { sinon }\end{array}\right.$

$\mathrm{B}=\sum_{u=\max (0, h+(j-n)-l)}^{\min (h, h+i+(j-n)-l)}\left[\begin{array}{c}\left(C_{h}^{u} p(S \rightarrow I)^{u} p(S \rightarrow S)^{h-u}\right) \\ \left(C_{i}^{u+i-m} p(I \rightarrow S)^{u+i-m} p(I \rightarrow I)^{m-u}\right) \\ \left(C_{j}^{j-n} p(P \rightarrow S)^{j-n} p(P \rightarrow P)^{n}\right)\end{array}\right]$

A t0, la transition $T((h, i, j), a,(l, m, n))$ est donnée par

$$
\sum_{u l=0}^{h} \sum_{u 2=0}^{h-u l} \sum_{v=0}^{i} \sum_{w=0}^{j} \delta_{\begin{array}{c}
h+v+w-u l-u 2=l \\
i+u l-v=m \\
j-w+u 2=n
\end{array}}\left[\begin{array}{c}
\left(C_{h}^{u 1} C_{h-u 1}^{u 2} p(S \rightarrow I \mid a)^{u l} p(S \rightarrow P \mid a)^{u 2} p(S \rightarrow S \mid a)^{h-u l-u 2}\right) \\
\left(C_{i}^{v} P(I \rightarrow S \mid a)^{v} P(I \rightarrow I \mid a)^{i-v}\right) *\left(C_{j}^{w} p(P \rightarrow S \mid a)^{w} p(P \rightarrow P \mid a)^{j-w}\right)
\end{array}\right]
$$

où $u 1$ le nombre de troupeaux $\mathrm{S}$ devenant $\mathrm{I}, u 2$ le nombre de troupeaux $\mathrm{S}$ devenant $\mathrm{P}, v$ le nombre de troupeaux I devenant $\mathrm{S}$ et $w$ le nombre de troupeaux $\mathrm{P}$ devenant $\mathrm{S}$. 
Considérant que les états vérifient la fonction de Dirac, l'équation précédente se simplifie en :

$$
T((h, i, j), a,(l, m, n))=\left\{\begin{array}{l}
B \text { si }\left\{\begin{array}{l}
m \leq i+h \\
n \leq h+j \\
l \leq j-n+h+i
\end{array}\right. \\
0 \text { sinon }
\end{array}\right.
$$

avec

$$
\mathrm{B}=\sum_{u 2=\max (0, n)}^{\min (n, h-(m-i))}\left[\begin{array}{l}
\min (h-u 2, h+h+i+(j-n)-l l \\
\sum_{u l=\max (0, h+(j-n)-l)}\left[\begin{array}{l}
\left(C_{h}^{u 2} C_{h 1-u 2}^{u 1} p(S \rightarrow I \mid a)^{u 1} p(S \rightarrow P \mid a)^{u 2} p(S \rightarrow S \mid a)^{h-u l-u 2}\right) \\
*\left(C_{i}^{u l+i-m} p(I \rightarrow S \mid a)^{u l+i-m} p(I \rightarrow I \mid a)^{m-u I}\right) \\
*\left(C_{j}^{j+u 2-n} p(P \rightarrow S \mid a)^{j+u 2-n} p(P \rightarrow P \mid a)^{n-u 2}\right)
\end{array}\right]
\end{array}\right]
$$

Pour calculer les transitions $\mathrm{T}$ au niveau du groupe, nous utilisons les transitions $\mathrm{P}(\mathrm{I} \rightarrow \mathrm{S}), \mathrm{P}(\mathrm{P} \rightarrow \mathrm{S}), \mathrm{P}(\mathrm{S} \rightarrow \mathrm{P})$ et $\mathrm{P}(\mathrm{S} \rightarrow \mathrm{I})$ définies dans le tableau 1 avec la particularité que si $\mathrm{t} \neq \mathrm{t} 0, \mathrm{P}(\mathrm{S}->\mathrm{P})=0$ quelque soit l'action et $\mathrm{si} \mathrm{t}=\mathrm{t} 0, \mathrm{P}(\mathrm{S}->\mathrm{P})$ prend des valeurs différentes selon la décision du collectif (ne rien faire ou recommander).

\subsubsection{Les récompenses}

Les récompenses sont définies au niveau de la structure. Elles comprennent un coût lié à la maladie $(\mathrm{Cm})$ par troupeau dans l'état individuel I. Lorsqu'il y a une recommandation à $\mathrm{t} 0$, le coût de la protection $(\mathrm{Cp})$ est considéré pour tous les troupeaux qui sont $\mathrm{P}$ après l'action. A t0, si le collectif ne fait rien, certains troupeaux sont quand même protégés (transition $\mathrm{S}$ à $\mathrm{P}$ possible) car chaque éleveur décide individuellement d'appliquer la protection ou non. On suppose donc à t0 que tous les troupeaux qui sont $\mathrm{P}$ suite à la décision du collectif ont été protégés induisant un coût de la protection $(\mathrm{Cp})$. Lorsque l'action Recommander est retenue, un coût fixe $(\mathrm{Cf})$ est ajouté. On a donc les formules suivantes :

$$
\left\{\begin{array}{c}
R\left(\left[(h, i, j), t_{0}\right], \text { Ne rien Faire, }\left[(k, l, m), t_{l}\right]\right)=C m * i+C p * m \\
R\left(\left[(h, i, j), t_{0}\right], \text { Recommander },\left[(k, l, m), t_{l}\right]\right)=C m * i+C p * m+C f \\
R\left(\left[(h, i, j), t_{i}\right], \text { Ne rien Faire, }\left[(k, l, m), t_{i+l}\right]\right)=C m * i \quad t_{i} \neq t_{0}
\end{array}\right.
$$

\subsubsection{Résolution et évaluation}

La politique est calculée en utilisant l'algorithme Value Iteration (Puterman, 2005) avec un horizon infini et un facteur d'atténuation mensuel de 0,997 (correspondant à un taux d'actualisation d'environ 3\% par an). Pour la convergence de l'algorithme, nous avons fixé le seuil d'erreur (epsilon) à la valeur 0,1 . Cette valeur nous semble acceptable étant donné que les valeurs obtenues pour les états varient entre -28000 et -32000 . 
Pour évaluer l'effet de la politique sur la propagation de la maladie dans une structure, nous avons simulé cette propagation sans aucune recommandation puis en utilisant la politique calculée. Nous avons considéré une structure de $\mathrm{N}=100$ troupeaux avec comme situation initiale l'état stable du modèle sans recommandation: 77 troupeaux infectés, 4 troupeaux protégés, 9 troupeaux sensibles. Comme notre modèle est stochastique, 10000 répétitions ont été réalisées pour décrire la variabilité de la propagation de la maladie dans la structure. Comme nous avons considéré un horizon infini pour la détermination de la politique, nous avons simulé la propagation sur 50 ans pour avoir des résultats sur une période assez longue.

La résolution du MDP et la simulation du modèle ont été programmées en Java (Flanagan, 2005).

\subsection{Résultats}

\subsubsection{Calcul de la politique}

La politique calculée lors de la résolution du MDP retient la recommandation dans un grand nombre d'états (Figure 2). Néanmoins, quand le nombre de troupeaux sensibles (S) ou le nombre de troupeaux infectés (I) est très petit, la politique indique qu'il ne faut pas faire de recommandation. On a donc une politique adaptative selon la situation épidémiologique dans le groupe.

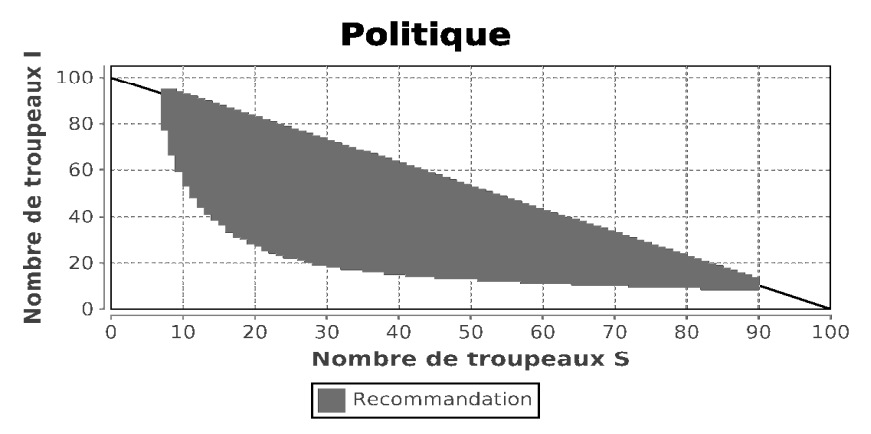

Figure 2. Politique MDP calculée en ti=t0 avec un horizon infini et un facteur d'atténuation de 0,997 (correspondant à un taux d'actualisation de 3\% par an)Représentation des couples (S, I) pour lesquels l'action « Recommandation » est retenue, où $S$ est le nombre de troupeaux sensibles et I le nombre de troupeaux infectés. 


\subsubsection{Qualité de la politique}

Pour l'évaluation de la qualité de la politique, on compare l'effet de la politique à celle où aucune recommandation n'est retenue et à celle où la recommandation est systématique. Les 5 premières années, la politique conduit à faire une recommandation dans plus de $90 \%$ des simulations avec la même situation initiale (Figure 3). L'utilisation de la recommandation quand on suit la politique décroît très lentement avec le temps et reste toujours supérieure à $60 \%$. Bien que l'objectif de l'optimisation porte sur les coûts, la politique MDP utilise l'action coûteuse « recommander », réduisant ainsi la prévalence de la maladie dans le groupe (figure 4). L'utilisation de la politique MDP conduit à une prévalence au bout de 20 ans (240 mois) inférieure ou égale à la prévalence obtenue si on ne fait aucune recommandation ( $\mathrm{Ne}$ rien faire). La politique MDP ne permet pas d'avoir une éradication de la maladie dans le groupe contrairement à l'utilisation systématique de la recommandation (Figure 4).

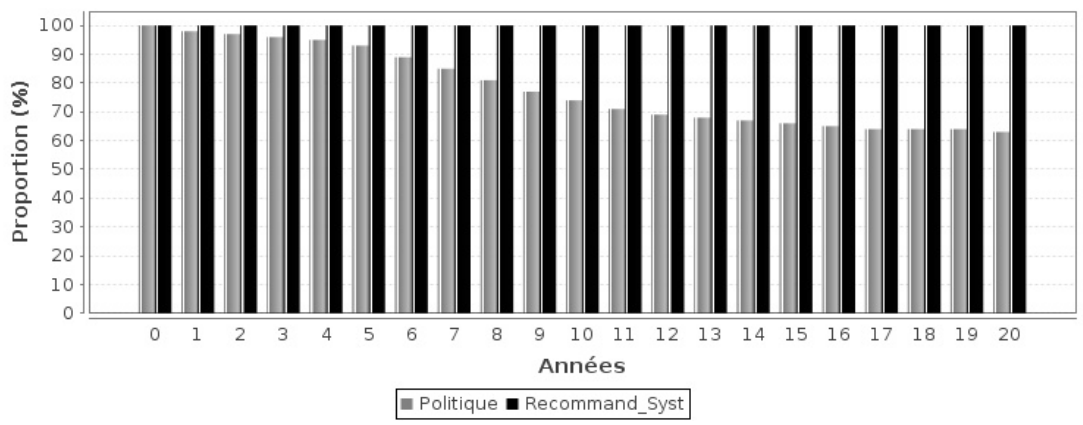

Figure 3. Proportion de simulations où l'action Recommandation est retenue dans les simulations lorsqu'on utilise la politique MDP (gris) ou une recommandation systématique (noir) en partant à l'année 0 d'une prévalence de $77 \%$ de troupeaux infectés.

Sur le long terme (50 ans), l'utilisation de la politique conduit bien à un coût total actualisé souvent plus faible que ceux en cas de recommandation systématique ou sans recommandation (Figure 5). Dans plus de 75\% des simulations, les coûts totaux au bout de 50 ans pour la politique MDP sont inférieurs à ceux des autres stratégies. En revanche, 20 ans après le début d'utilisation de la politique, les coûts sont parfois légèrement supérieurs à ceux de la politique sans recommandation, mais restent souvent inférieurs à ceux obtenus en recommandant systématiquement. 


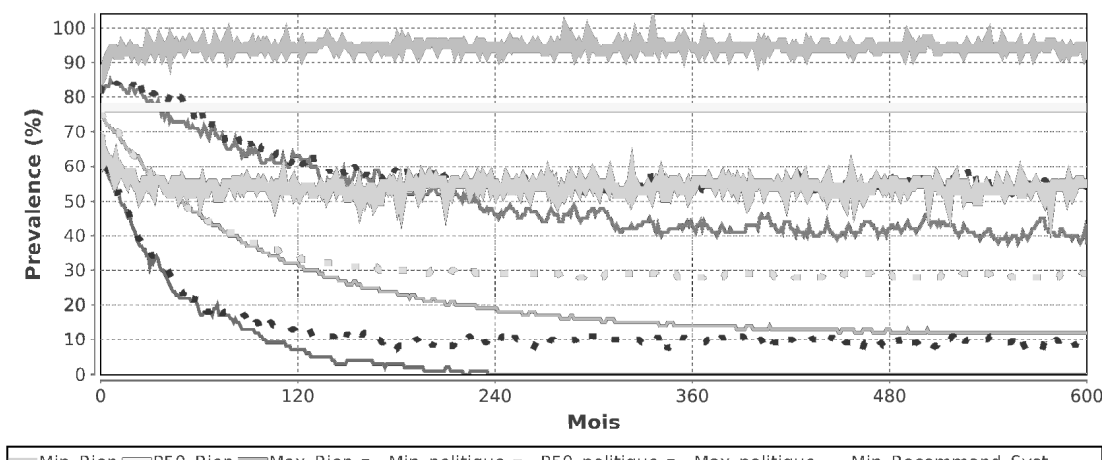

Min_Rien $\square$ P50_Rien Max_Rien - Min_politique a P50_politique - Max_politique — Min_Recommand_Syst - P50 Recommand Syst $=$ Max Recommand Syst

Figure 4. Evolution de la prévalence des troupeaux infectés (I) dans le groupe lorsqu'on utilise la politique MDP (_Politique), quand on retient toujours l'action Ne Rien Faire (_Rien) et avec une recommandation systématique (_Recommand_Syst) en partant à l'année 0 d'une prévalence de $77 \%$ de troupeaux infectés. Description par la prévalence médiane (P50), minimale (Min) et maximale (Max)
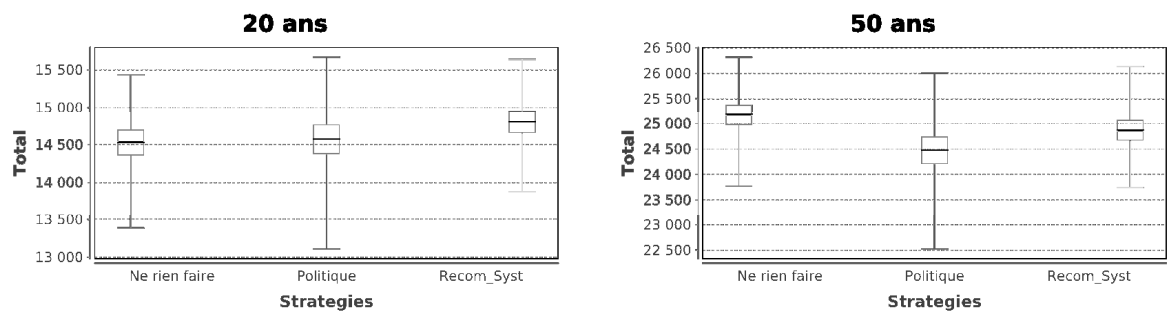

Figure 5. Boxplot des coûts totaux actualisés à un taux correspondant à $3 \%$ par an, au bout de 20 et 50 ans en utilisant la politique MDP (Politique), sans recommandation (Ne rien faire) et avec recommandation systématique (Recom_Syst), en partant à l'année 0 d'une prévalence de $77 \%$ de troupeaux infectés.

\subsubsection{Effet du suivi de la recommandation sur la politique}

Le nombre d'états du modèle où la recommandation est retenue augmente quand la probabilité de suivi de la recommandation augmente (Figure 6), et inversement. Lorsque la recommandation n'induit pas plus de prévention que si le collectif choisit de ne rien faire, la politique retient toujours l'action « Ne rien faire » du fait des coûts fixes de recommandation applicables pour le collectif. En revanche, si on modifie la valeur de la probabilité de passer de $\mathrm{S}$ à $\mathrm{P}$ pour représenter un scénario où 
la recommandation serait suivie par quasiment tous les éleveurs (99\% de protection quand le collectif fait des recommandations), on n'obtient pas une recommandation systématique dans la politique optimale (Figure 6). En effet, il n'est pas intéressant de recommander la protection lorsque les troupeaux sont presque tous sains, protégés, ou infectés.

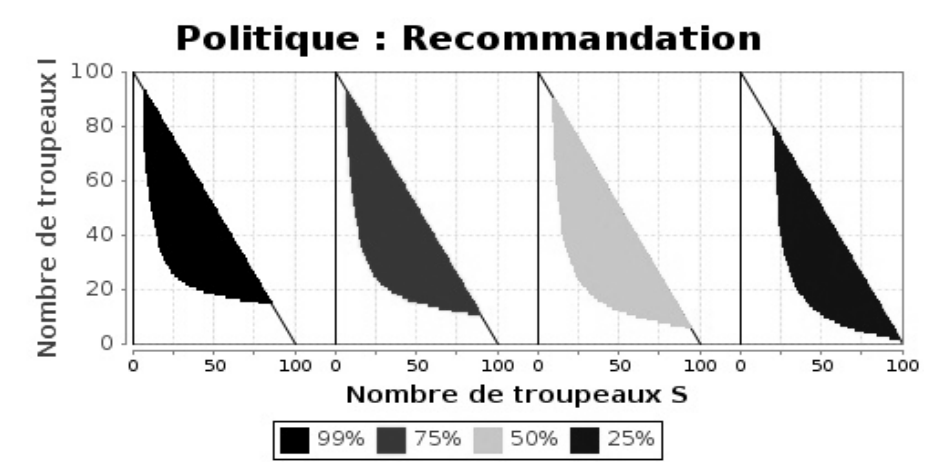

Figure 6. Effet de la valeur de la probabilité de passer de $S$ à $P$ suite à la recommandation sur la politique calculée par le MDP avec un horizon infini et un facteur d'atténuation de 0.997 - Représentation des couples $(S, I)$ pour lesquels l'action " recommandation » est retenue où $S$ est le nombre de troupeaux sensibles et I le nombre de troupeaux infectieux.

\section{Discussion et conclusion}

L'utilisation d'un MDP intégrant un modèle épidémiologique représentant une maladie endémique permet de choisir de façon optimale à chaque pas de temps s'il faut recommander ou non la mise en place une action de prévention selon la situation courante. La définition de cette stratégie qui s'adapte selon la situation épidémiologique est un apport des approches d'optimisation, en plus de la garantie d'optimalité. Le modèle permet de proposer différentes politiques en fonction des objectifs à optimiser. Même si la politique obtenue est stationnaire, l'action retenue à chaque instant dépendra de la situation épidémiologique courante. La séquence de décision (recommander ou pas) finalement retenue variera au cours du temps pour s'adapter à l'état du processus. Dans notre exemple, la recommandation n'est pas retenue dans toutes les simulations, elle s'adapte à l'évolution de la propagation. Notre modèle peut être étendu très facilement pour considérer d'autres actions possibles (par exemple limitation des échanges d'animaux entre troupeaux selon leur statut).

Dans ce papier, nous utilisons une approche classique permettant de résoudre des problèmes de prises de décision séquentielle qui est aussi utilisée pour la gestion de population. Nous considérons ici un groupe d'élevages avec un décideur unique. 
L'utilisation d'un MDP permettant de proposer une séquence d'actions pour atteindre un objectif dans une méta-population a déjà été réalisée en écologie pour répondre à des questions liées à la conservation d'espèce ou de biodiversité (Possingham, 1996; Chadès et al., 2008). La dynamique de transmission entre troupeaux pourrait être représentée avec une approche de méta-population intégrant les mouvements d'animaux entre troupeaux (tel que dans Le Menach et al., 2006). Comme dans McDonald-Madden et al. (2008), nous ne considérons pas explicitement les mouvements d'animaux. Néanmoins, dans notre modèle, les troupeaux sont en interaction du fait de la propagation de l'agent pathogène bien que nous n'ayons pas défini de réseaux de contacts liés aux échanges d'animaux. Pour définir ces réseaux, des données sur les mouvements d'animaux (tels que dans Ezanno et al., 2006; Ribbens et al., 2009) sont nécessaires. L'introduction de ce réseau dans un MDP va augmenter la taille du modèle et donc rendre plus complexe sa résolution. Pour l'optimisation, un Graph-based-MDP (Peyrard et al., 2007 ; Sabbadin et al., 2012) pourrait alors être envisagé. Néanmoins, même si on gagne de la précision sur la modélisation de la transmission, la résolution ne sera possible que de manière approchée. En santé humaine, l'impact des décisions individuelles sur la propagation est souvent étudié (par exemple : Durham et Casman , 2012 ; Mbah et al., 2012) mais l'étude de recommandations pré-définies pour coordonner des décisions individuelles explicitement représentées est moins fréquente (Manfredi et al., 2009 ; Althouse et al., 2010) et souvent évaluée par simulation.

Le MDP est basé sur l'hypothèse de Markov. Cette hypothèse suppose que la transition dépend uniquement de l'état courant. Il est possible de définir des états incluant des éléments d'historiques nécessaires pour définir correctement les transitions, par exemple pour représenter des transitions semi-Markoviennes. Dans notre modèle, nous avons eu recours à cette étape pour représenter le fait que la décision a lieu tous les ans donc tous les 12 pas de temps. On a donc introduit dans le modèle une composante représentant le mois de l'année pour ne calculer la décision que tous les ans à la même date. Un procédé similaire peut être utilisé pour représenter un délai pour avoir un effet complet de la protection. Néanmoins, le rajout de composantes ou de statuts individuels va augmenter la taille du MDP et donc rendre plus difficile sa résolution. Le choix des états est donc à faire en gardant en tête ces limitations.

Comme nous avons modélisé des mesures de protections prises une seule fois par an, notre modèle aurait pu être défini avec un pas de temps annuel. Néanmoins, nous avons choisi de présenter le modèle avec un pas de temps de 1 mois pour pouvoir intégrer ultérieurement d'autres actions. En effet, on peut imaginer qu'une action puisse avoir des impacts et des coûts différents au cours du temps au regard de la gestion du troupeau. Par exemple, les mesures de biosécurité pourraient être retenues que certains mois, correspondant par exemple à la période en pâturage. De plus, les mesures de protection pourraient avoir des coûts et des conséquences différentes selon si les animaux sont en pâture ou en bâtiment. Introduire des mesures de biosécurité différentes selon les mois est possible avec notre modèle. Pour simplifier la présentation du modèle, nous avons considéré une action 
disponible une fois par an à la même date tous les ans. Néanmoins, notre modèle pourrait facilement être étendu à des situations où les décisions de protection doivent être séparées d'au moins 12 mois. Dans ce cas, la transition de t0 à t1 ne sera possible qu'en cas d'action de protection. Si l'action « Ne rien faire » est retenue en t0, la transition sera de $\mathrm{t} 0$ à $\mathrm{t} 0$. On aura alors bien un écart de minimum 12 mois mais l'action ne sera pas réalisée à la même date tous les ans.

Le choix de l'horizon pour l'optimisation est complexe. Si l'objectif est à optimiser sur du très long terme, l'horizon infini est le plus simple. Néanmoins, les décideurs prennent souvent des objectifs à optimiser sur un horizon fini. Cet horizon est souvent glissant, intégrant chaque année les informations supplémentaires pour revoir leur prise de décision : par exemple l'évolution de la prévalence ou des coûts du marché. On est alors dans une logique d'horizon glissant. Ce type d'horizon a été proposé et utilisé par plusieurs auteurs (Millar et al., 1998 ; Van der Broek , 2002 ; Balakrishnan et al., 2009 ; Mosnier et al., 2009). Il consiste à calculer tous les ans la politique à horizon fini $h$ et de n'utiliser que la politique correspondant à $h$ années avant la fin. Si $h$ est assez grand, cette politique sera proche de celle avec un horizon infini. Cette politique diffère d'une politique MDP à horizon fini dans le sens où l'exécution de la politique ne tient pas compte du temps restant avant la fin de l'horizon. En effet, une politique pour un horizon fini est, en général, non stationnaire. Si $h$ est l'horizon et $\pi_{n}$ est la politique à appliquer quand il reste $n$ décisions à prendre avant d'atteindre la fin de l'horizon, $\pi_{0}$ est généralement différente de $\pi_{1}$ elle même différente de $\pi_{2}$, etc. L'idée de la fenêtre glissante est, à chaque instant, de recalculer les $\pi_{i}$, et d'appliquer $\pi_{h}$. A l'instant $t$, la valeur de politique consistant à toujours recalculer $\pi_{h}$ pour l'appliquer est différente de la valeur que l'on aurait en utilisant effectivement les politiques $\pi_{h}, \pi_{h-1}, \pi_{h-2}, \ldots$, qui tiennent compte du temps restant avant la fin de l'horizon.

L'objectif pour l'optimisation peut être très variable. Dans notre exemple, nous avons retenu une minimisation des coûts. On pourrait aussi bien rechercher une minimisation de la prévalence, ou considérer un seuil de prévalence à atteindre à moindre coût. La formalisation sous forme de récompenses dans le modèle serait différente mais ne changerait en rien la résolution du modèle. En revanche, si on souhaite avoir un objectif multi-critères, une approche du type 2V-MDP (Boussard, 2008) est à envisager. La complexité de résolution de ce type de modèle est la même que celle d'un MDP.

Lorsque le nombre de troupeaux ou le nombre de statuts individuels augmente, la taille de l'espace d'état augmente de manière exponentielle. La résolution du MDP peut être rendue très longue voire impossible selon la capacité mémoire des calculateurs utilisés. Une solution possible consiste à passer d'un nombre de troupeaux à une proportion de troupeaux. Les états deviennent alors continus. Ce nouveau modèle peut être résolu à l'aide d'approximateurs de fonctions (Munos, 
2008 ; Sherrer et Thiery, 2009) ou en discrétisant les proportions (Yaesoubi et Cohen, 2011) ce qui se ramène au modèle que nous avons présenté. Il est difficile de trouver un bon compromis entre une discrétisation fine proche du modèle original mais complexe à résoudre et une approximation grossière facile à résoudre.

Dans cet article, le modèle fait des hypothèses très simplistes sur la maladie représentée et sur les connaissances du décideur collectif. Les trois statuts individuels considérés pour l'instant ne seront pas suffisants pour décrire la propagation de certaines maladies. Par exemple, pour des maladies induisant une immunité du troupeau après infection, un état supplémentaire doit être introduit dans le MDP individuel. Dans le cas où une maladie reste inapparente pendant plusieurs années, par exemple comme la paratuberculose bovine (Manning et Collins, 2001), il faut introduire là aussi un état supplémentaire mais qui est non observable. Un MDP ne peut donc plus être défini car ce type de modèle suppose une observabilité totale du système. Une approche par MDP avec observation partielle (POMDP) est envisageable (Kaebling et al., 1998). Ce même type de modèle peut aussi être utilisé si on ne connaît pas exactement le statut des troupeaux, ce qui est souvent le cas car la sensibilité des tests de dépistage est rarement de 100\% (Christensen et al., 2000). Les POMDP ont néanmoins un désavantage lié à leur résolution complexe qui est réalisée le plus souvent de manière approchée.

Notre modèle est un outil d'aide à la prise de décision. Cet outil peut servir à simuler plusieurs scénarios pour explorer les politiques obtenues selon les valeurs des probabilités de transitions (sans et avec recommandation). Pour une maladie réelle, le plus difficile est d'estimer les probabilités de transitions avec ou sans recommandation. En santé humaine, Chapman et al. (2012) explorent par expérimentation («game-theory experiments ») le comportement d'un échantillon de personnes. En santé animale, les organisations collectives ont parfois déjà mis en œuvre pour d'autres maladies des recommandations (ou plans de maîtrise) ce qui peut les aider à définir les probabilités de transitions sans et avec recommandations. L'impact des incertitudes pour ces valeurs peut être étudié par des méthodes d'analyse de sensibilité (Saltelli et al., 2000). L'apprentissage par renforcement (Sutton et Barto, 1998) permettrait aussi de définir progressivement le modèle mais nécessite une phase d'apprentissage dont on ne dispose pas toujours. Il serait aussi intéressant de tester des hypothèses faites dans le modèle. En particulier, Rat-Aspert et Krebs (2012) ont montré par modélisation que les décisions individuelles pouvaient varier selon la prévalence de l'infection dans le groupe. Si pour une maladie d'intérêt, ce type de comportement est observé, il faudrait alors faire varier les probabilités de transitions de $\mathrm{S}$ à $\mathrm{P}$ selon la prévalence ce qui est possible dans notre modèle sans en augmenter la complexité.

Dans ce modèle, nous avons simplifié le problème pour s'affranchir de la représentation séparée des prises de décision individuelles nous permettant de limiter la taille du problème à résoudre. Bien que simplifié notre problème permet au décideur collectif de réfléchir en terme de stratégie non systématique. Néanmoins, les interactions entre décisions individuelles et collectives pourraient être considérées de manière plus précises, nécessitant de représenter explicitement les 
prises de décisions individuelles. L'étape suivante sera donc d'intégrer dans le modèle des décisions hiérarchiquement imbriquées. Les approches MDP multi-agent (MMDP, Boutilier, 1999) ou MDP décentralisé (Dec-MDP, Bernstein et al., 2002) permettent de tenir compte des actions individuelles. Néanmoins, leur résolution ne peut être réalisée que pour un petit nombre d'élevages. Pour modéliser ce problème, des approches du type Leader-Follower (Tharakunnel et Bhattacharyya, 2009; Sabbadin et Viet, 2013) ou multi-échelles (Wernz et Deshmukh, 2010, 2012) ou principal-agent dynamique (Plambeck et Zenios, 2000) peuvent être envisagées.

\section{Remerciements :}

Ce travail a bénéficié d'un financement de l'INRA, du Cemagref et des Conseils Régionaux de Basse-Normandie, Bretagne, Pays de le Loire et Poitou-Charentes, au titre du projet SANCRE, dans le cadre des programmes PSDR et d'une aide de l'Etat gérée par l'Agence Nationale de la Recherche au titre du programme Investissements d'avenir portant la référence ANR-10-BINF-07 (MIHMES).

\section{Bibliographie non numérotée et références}

Althouse B. M., Bergstrom T. C., Bergstrom C. T. (2010). A public choice framework for controlling transmissible and evolving diseases. Proceeding of the National Academy of Sciences, vol. 107, p. 1696-1701.

Balakrishnan J., Hung Cheng C. (2009). The dynamic plant layout problem: Incorporating rolling horizons and forecast uncertainty. Omega, vol. 37, p. 165-177.

Backer J.A., Hagenaars T.J., van Roermund H.J.W., de Jong M.C.M. (2009). Modelling the effectiveness and risks of vaccination strategies to control classical swine fever epidemics. Journal of the Royal Society Interface, vol. 6, p. 849-861.

Begon M, Bennett M, Bower RG, French NP, Hazel SM, Turner J. (2002). A clarification of transmission terms in host-microparasite models: numbers, densities and areas. Epidemiology and Infection, vol. 129, p. 147-153.

Bernstein D.S., Givan R., Immerman N., Zilberstein S. (2002). The complexity of decentralized control of Markov Decision Processes. Mathematical Operation Research, vol. 27 , p. $819-840$.

Boussard M. (2008). Planification multi-agents multi-objectifs : modèle et algorithme. Doctorat spécialité Informatique, Université Caen Basse-Normandie.

Boussard M., Bouzid M., Mouaddib A.I. (2007). MultiCriteria decision Making for local coordination. Actes de Annual IEEE International Conference on Tools with Artificial Intelligence (ICTAI), Grèce, Patras.

Boutilier, C. (1999). Sequential optimality and coordination in multi-agent systems. In: Proceedings of the 16th International Joint Conference on Artificial Intelligence, pages 478-485. 
Christensen J., Gardner I.A. (2000). Herd-level interpretation of test results for epidemiologic studies of animal diseases. Preventive Veterinary Medicine, vol. 45, p. 83-106.

Chadès I ., McDonald-Madden E., McCarthy M.A., Wintle B., Linkie M., Possingham H.P. (2008). When to stop managing or surveying cryptic threatened species. Proceeding of the National Academy of Sciences, vol. 105, p. 13936-13940.

Chadès I, Martin TG, Burgman MA, Possingham HP, Buckley YM. (2011). General rules for managing and surveying networks of pests, diseases, and endangered species. Proceeding of the National Academy of Sciences, vol. 108, p. 8323-8328.

Chapman G. B., Li M., Vietri J., Ibuka Y., Thomas D., Yoon H., Galvani A. P. (2012). Using game theory to examine incentives in influenza vaccination behavior. Psychological Science, vol. 23, p. 1008-1015

Durham D.P., Casman E.A. (2012). Incorporating individual health-protective decisions into disease transmission models: a mathematical framework. Journal of The Royal Society Interface, vol. 9 , p. 562-570

Ezanno P., Fourichon C., Beaudeau F., Seegers H. (2006). Between-herd movements of cattle as a tool for evaluating the risk of introducing infected animals. Animal Research, vol. 55, p. $189-208$.

Ferguson N.M., Donnelly C.A., Anderson R.M. (2001). The Foot-and-Mouth epidemic in Great-Britain: Pattern of spread and impact of interventions. Science, vol. 292, p. 11551160.

Flanagan D. (2005), Java in a nutshell, Fifth Edition. O'Reilly Media.

Forsell N., Wikström P, Garcia F., Sabbadin R., Blennow K., Eriksson L.O. (2011). Management of the risk of wind damage in forestry: a graph-based Markov decision process approach. Annals of Operations Research, vol. 190, p. 57-74.

Galvani A.P., Reluga T.C., Chapman G.B. (2007). Long-standing influenza vaccination policy is in accord with individual self-interest but not with the utilitarian optimum. Proceeding of the National Academy of Sciences, vol. 104, p.5692-5697.

Garcia F. (2008). Processus Décisionnels de Markov. Processus décisionnels de Markov en intelligence artificielle - volume 1 - principes généraux et applications. Paris, Lavoisier, p. 19-52.

Ge L, Kristensen AR, Mourits MC, Huirne RB. (2010). A new decision support framework for managing foot-and-mouth disease epidemics. Annals of Operations Research, In press.

Johnson F, Breininger D, Duncan BW, Nichols JD, Runge MC, Williams BK. (2011). A Markov Decision Process for Managing Habitat for Florida Scrub-Jays. Journal of Fish Wildlife Management, vol. 2, p. 234-246.

Kaelbling L.P., Littman M.L., Cassandra A.R. (1998). Planning and acting in partially observable stochastic domains. Artificial Intelligence, vol. 101, p. 99-134.

Keeling M.J., Woolhouse M.E.J., May R.M., Davies G., Grenfell B.T. (2003). Modelling vaccination strategies against foot-and-mouth disease. Nature, vol. 421, p. 136-142. 
Klein E., Laxminaryan R., Smith D.L., Gilligan C.A. (2007). Economic incentives and mathematical models of disease. Environment and Development Economics, vol. 12, p. 707-732

Kobayashi M., Carpenter T.E., Dickey B.F., Howitt R.E. (2007). A dynamic, optimal disease control model for foot-and-mouth disease:: I. Model description. Preventive Veterinary Medicine, vol. 79, p. 257-273.

Kristensen A.R. (2003). A general software system for Markov decision processes in herd management applications. Computer and Electronics in Agriculture, vol. 38, p. 199-215

Le Menach A., Vergu E., Grais R.F., Smith D.L., Flahault A. (2006). Key strategies for reducing spread of avian influenza among commercial poultry holdings: lessons for transmission to humans. Proceedings of the Royal Society of London B - Biological Science, vol. 273, p. 2467-2475.

Manfredi P., Posta P. D., d'Onofrio A., Salinelli E., Centrone F., Meo C., Poletti P. (2009). Optimal vaccination choice, vaccination games, and rational exemption: An appraisal. Vaccine, vol 28, p. 98-109.

Manning E.J., Collins M.T. (2001). Mycobacterium avium subsp. Paratuberculosis: pathogen, pathogenesis and diagnosis, Revue Scientifique et Technique de l'OIE, vol. 20, p. 133150.

Mbah M.L.N., Liu J., Bauch C.T., Tekel Y.I., Medlock J., Meyers L.A., Galvani A.P. (2012). The impact of imitation on vaccination behavior in social contact networks. PLoS computational biology, vol. 8, e1002469.

McDonald-Madden E., Baxter P.W.J., Possingham H.P. (2008). Subpopulation triage: How to allocate conservation effort among populations. Conservation Biology, vol. 22, p. 656665 .

Merl D., Johnson L.R., Gramacy R.B., Mangel M.A. (2009). Statistical Framework for the Adaptive Management of Epidemiological Interventions. PLoS ONE, vol. 4, e5807.

Millar H.H. (1998). The impact of rolling horizon planning on the cost of industrial fishing activity. Computers and Operations Research, vol. 25, p. 825-837.

Mosnier C., Agabriel J., Lherm M., Reynaud A. (2009). A dynamic bio-economic model to simulate optimal adjustments of suckler cow farm management to production and market shocks in France. Agricultual Systems, vol. 102, p. 77-88.

Moore CT, Fonnesbeck JF, Shea K, Lah KJ, McKenzie PM, Ball LC, Runge MC, Alexander HM. (2011). An adaptive decision framework for the conservation of a threatened plant. Journal of Fish Wildlife Management, vol. 2, p. 247-261.

Munos R. (2008). Programmation dynamique avec approximation de la fonction valeur. In $\mathrm{O}$. Sigaud, O. Buffet (eds), Processus décisionnels de Markov et intelligence artificielle, volume 2, chapter 11, pages 19-50.

Nöremark M., Håkansson N., Lewerin S.S., Lindberg A., Jonsson A. (2011). Network analysis of cattle and pig movements in Sweden: Measures relevant for disease control and risk based surveillance. Preventive veterinary medicine, vol. 99, p. 78-90.

Peyrard N., Sabbadin R., Aubertot J.N. (2007). A graph-based markov decision process framework for optimising integrated management of diseases in agriculture. Actes du 
17th Inter. Congress on Modelling and Simulation (MODSIM'07), New-Zealand, Christchurch.

Plambeck E.L., Zenios S.A. (2000). Performance-based incentives in a dynamic PrincipalAgent model. Manufacturing and Service Operations Management, vol. 2, p. 240-263.

Possingham H. P. (1996). Decision theory and biodiversity management: how to manage a metapopulation. In Frontiers of Population Ecology. CSIRO, Melbourne, p. 391-398

Puterman M.L. (2005). Markov decision processes: Discrete stochastic dynamic programming. John Wiley \& Sons Inc.

Rat-Aspert O., Krebs S. (2012). Individual and collective management of endemic animal diseases: an economic approach. In : Proceeedings of the IAAE (International Association of Agricultural Economists) conference, Foz do Iguacu, Brazil.

Rat-Aspert O., Fourichon C. (2010). Modelling collective effectiveness of voluntary vaccination with and without incentives. Preventive Veterinary Medicine, vol. 93, p. 265275.

Ribbens S., Dewulf J., Koenen F., Mintiens K., de Kruif A., Maes D. (2009). Type and frequency of contacts between Belgian pig herds. Preventive Veterinary Medicine, vol. 88 , p. $57-66$.

Sabbadin R., Viet A.-F. (2013). A tractable Leader-Follower MDP model for animal disease management. In : 27th AAAI conference (Association for the advancement of artificial intelligence), Bellevue (Washington), USA.

Sabbadin R., Peyrard N., Forsell N. (2012). A framework and a mean-field algorithm for the local control of spatial processes. International Journal of Approximate Reasoning, vol. 53, p. 66-86.

Saltelli A., Chan K., E. Scott (2000). Sensitivity analysis. John Wiley and Sons, ldt.

Scherrer B, Thiéry C. (2009). Construction d'un joueur artificiel pour Tetris. Revue d'Intelligence Artificielle, vol. 23, p. 387-407.

Seegers H., Billon D., Grimard B., Beaudeau F. (2006). A Simulation Model To Study Reproductive Disorders And Reproductive Management In Dairy Herds. In, 11th International Symposium on Veterinary Epidemiology and Economics, Cairns, Australie.

Sutton RS, Barto AG (1998). Reinforcement learning: an introduction. Adaptive Computation and Machine Learning series. MIT Press, Cambridge, Mass.

Tharakunnel K., Bhattacharyya S. (2009). Single-leader-multiple-follower games with boundedly rational agents. Journal of Economic Dynamics \& Control, vol. 33, p. 15931603.

Thrusfield M. (1986). Veterinary epidemiology. Butterworths, London

Tildesley M.J., Savill N.J., Shaw D.J., Deardon R., Brooks S.P., Woolhouse M.E.J., Grenfell B.T., Keeling M.J. (2006). Optimal reactive vaccination strategies for a foot-and-mouth outbreak in the UK. Nature, vol. 440, p. 83-86.

Toft N., Kristensen A.R., Jørgensen E. (2005). A framework for decision support related to infectious diseases in slaughter pig fattening units. Agricultural Systems, vol. 85, p. 120137. 
van den Broek W.A. (2002). Moving horizon control in dynamic games. Journal of Economic Dynamics \& control, vol. 26, p. 937-961.

Viet A.F., Jeanpierre L., Bouzid M., Mouaddib A.-I. (2012). Using Markov Decision Process to define an adaptive strategy to control the spread of an animal disease. Computers and Electronics in Agriculture, vol. 80, p 71-79.

Wernz C., Deshmukh A. (2010). Multiscale decision-making: Briging organizational scales in systems with distributed decision-makers. Journal of Operational Research, vol. 202, p 828-840.

Wernz C., Deshmukh A. (2012). Unifying temporal and organizational scales in multiscale decision-making. European Journal of Operational Research, vol. 223, p. 739-751.

Yaesoubi R., Cohen T. (2011). Generalized Markov models of infectious disease spread: A novel framework for developing dynamic health policies. European Journal of Operation Research vol. 215, p 679-687.

Article reçu le : AR_1religne_soumission

Article accepté le : AR_soumission 